\title{
Agenda 2030, saúde e sistemas alimentares em tempos de sindemia: da vulnerabilização à transformação necessária
}

\author{
2030 Agenda, health and food systems in times of syndemics: \\ from vulnerabilities to necessary changes
}

André Campos Burigo (http://orcid.org/0000-0002-1640-6289) ${ }^{1}$

Marcelo Firpo Porto (https://orcid.org/0000-0002-9007-0584) ${ }^{2}$

\footnotetext{
${ }^{1}$ Vice-presidência de Ambiente, Atenção e Promoção da Saúde, Fundação Oswaldo Cruz. Av. Brasil 4.365, Manguinhos. 21040-900 Rio de Janeiro RJ Brasil. andreburigo@gmail.com ${ }^{2}$ Núcleo Ecologias, Epistemologias e Promoção Emancipatória da Saúde, Escola Nacional de Saúde Pública Sergio Arouca, Fundação Oswaldo Cruz. Rio de Janeiro RJ Brasil.
}

\begin{abstract}
This article, an essay, and narrative review, analyzes the relationship between the 2030 Agenda, food systems, and their relevance to global and collective health. The concept of syndemics contextualizes the COVID-19 pandemic in relation to poverty and social injustice, as it also reveals the synergy with other pandemics related to the advancement of the global food system: malnutrition, obesity, and climate change, which all have strong influence of the dominant model of agriculture. We also use four strategic concepts to think about the transition towards healthy and sustainable food systems: food system, food and nutrition security (FNS), human right to adequate food (HRAF) and agroecology. Then, we gather international reports and data that systematize studies on the growing threats imposed by the dominant agricultural model, often denied by powerful economic sectors and neoconservative groups. We also highlight challenges imposed at different scales, from global to local, so that public policies and social mobilizations developed in the last two decades can resist and reinvent themselves in the construction of fairer societies.

Key words Sustainable development, Diet, food, and nutrition, Health promotion, Sustainable agriculture, Agroecology
\end{abstract}

Resumo $O$ artigo, uma mescla de ensaio e revisão narrativa, analisa a relação entre a Agenda 2030, os sistemas alimentares e sua relevância para a saúde global e coletiva. O conceito de sindemia contextualiza a pandemia de COVID-19 em relação com a pobreza e com a injustiça social, mas também revela a sinergia com outras pandemias relacionadas ao avanço do sistema alimentar global: de desnutrição, de obesidade e das mudanças climáticas, as quais possuem forte influência do modelo dominante de agricultura. Lançamos mão, também, de quatro conceitos estratégicos para pensar a transição em direção a sistemas alimentares saudáveis e sustentáveis: sistema alimentar, segurança alimentar e nutricional (SAN), direito humano à alimentação adequada (DHAA) e agroecologia. Em seguida, cotejamos relatórios e dados internacionais que sistematizam estudos sobre as crescentes ameaças decorrentes do modelo dominante de agricultura, frequentemente negadas por setores econômicos poderosos e grupos neoconservadores. Também destacamos desafios colocados em diferentes escalas, do global ao local, para que políticas públicas e mobilizações sociais desenvolvidas nas últimas duas décadas possam resistir e se reinventar na construção de sociedades mais justas.

Palavras-chave Desenvolvimento sustentável, Alimento, dieta e nutrição, Promoção da saúde, Agricultura sustentável, Agroecologia 


\section{Introdução}

Este artigo, que mescla ensaio acadêmico e revisão narrativa, busca lançar luz sobre a importância estratégica dos sistemas alimentares para a promoção da saúde em diferentes dimensões e escalas, no diálogo com as grandes mudanças que vêm acontecendo no mundo e a Agenda de transformações necessárias, pactuadas a nível global (2015-2030).

Nesse sentido, a partir de reflexões sobre a Agenda 2030 em conexão com conceitos como saúde global e sindemia, busca-se evidenciar a importância dos sistemas alimentares agroecológicos, considerados simultaneamente sustentáveis e promotores de dietas saudáveis, para a promoção da saúde nas diferentes escalas: local, regional, nacional e global.

A perspectiva da Agenda 2030 também influencia e serve de importante alicerce à noção de Saúde Global. Esta pode ser considerada como uma questão emergente na Saúde Pública que congrega princípios ético-políticos e conhecimentos voltados para enfrentar iniquidades em saúde no mundo globalizado. Em termos acadêmicos, pode ser definida como uma área de caráter multiprofissional e interdisciplinar envolvendo questões e problemas de saúde supraterritoriais e multiescalares que extrapolam fronteiras geográfico-políticas nacionais. Seus determinantes sociais e ambientais podem ter origem em quaisquer lugares, assim como as suas possiveis soluções necessitam de intervenções e acordos entre diversos atores sociais, incluindo paises, governos $e$ instituiçães internacionais públicas e privadas (p.370) ${ }^{1}$.

Consideramos esse tema estratégico para o potencial avanço do conjunto dos Objetivos de Desenvolvimento Sustentável (ODS). Para tanto, apresenta-se ao longo do texto uma sistematização de conceitos considerados centrais e privilegia-se como referências e fontes de informações dez relatórios internacionais produzidos desde a pactuação da Agenda 2030. Os documentos selecionados apresentam sistematizações de literatura com análises sobre sistemas alimentares desde focos distintos, e foram produzidos por diferentes grupos internacionais de especialistas em segurança alimentar e nutricional, ou por especialistas em biodiversidade e mudanças climáticas a serviço de organizações vinculadas à ONU. Considera-se que a revisão desses relatórios contribui para mapear o debate atual sobre uma problemática ampla e complexa, levantando questões e colaborando para a atualização do conhecimento em temas ainda pouco trabalhados em publicações de artigos nos periódicos da saúde coletiva.

Os diversos estudos presentes nas referências selecionadas agregam um conjunto de evidências científicas que ajudam a delinear um entendimento da perigosa situação que o sistema alimentar dominante produz atualmente no mundo, mas também propiciam consistência argumentativa sobre a relevância e as alternativas que impulsionam outros sistemas alimentares, com destaque para a agroecologia. Por fim, ainda que de forma sintética, apontamos aspectos críticos da realidade brasileira, concluindo-se que, ao lado da gravidade dos retrocessos em andamento, também existem potencialidades expressas nas resistências e nos avanços em direção a sistemas alimentares agroecológicos.

\section{Agenda 2030: conexões entre saúde e alimentação em tempos de sindemia}

Na 70a Assembleia Geral da Nações Unidas (ONU), em setembro de 2015, representantes dos seus 193 Estados-membros se comprometeram com a Resolução "Transformando Nosso Mundo: A Agenda 2030 para o Desenvolvimento Sustentável". Reconheceram que a erradicação da pobreza em todas as suas formas é o maior desafio global e um requisito indispensável para o desenvolvimento sustentável - nas dimensões econômicas, social e ambiental ${ }^{2}$.

A Resolução tem respaldo em propósitos e princípios consagrados na Carta de fundação das Nações Unidas (1945), da Declaração Universal dos Direitos Humanos (1948), na Declaração do Rio sobre o Meio Ambiente (1992), na Declaração do Milênio (2000), na Declaração final da Conferência Rio+20 (2012), entre outros tratados e acordos internacionais. $\mathrm{O}$ contexto da Rio+20 e os Objetivos de Desenvolvimento do Milênio (2000-2015) serviram de base para a formulação da nova Agenda para os anos seguintes, considerada mais participativa e envolvendo dezenas de países e diversos setores das sociedades. A Agenda 2030 parte de um diagnóstico abrangente e incisivo dos desafios para a humanidade neste início do século XXI, concluindo que a sobrevivência de muitas sociedades e dos sistemas biológicos do planeta, está em risco²:

Bilhões de cidadãos continuam a viver na pobreza e a eles é negada uma vida digna. Há crescentes desigualdades dentro dos e entre os países. Há enormes disparidades de oportunidades, riqueza e poder. A desigualdade de gênero continua a ser um desafio fundamental. O desemprego, particu- 
larmente entre os jovens, é uma grande preocupação. Ameaças globais de saúde, desastres naturais mais frequentes e intensos, conflitos em ascensão, o extremismo violento, o terrorismo e as crises humanitárias relacionadas e o deslocamento forçado de pessoas ameaçam reverter grande parte do progresso do desenvolvimento feito nas últimas décadas. $O$ esgotamento dos recursos naturais e os impactos negativos da degradação ambiental, incluindo a desertificação, secas, a degradação dos solos, a escassez de água doce e a perda de biodiversidade acrescentam e exacerbam a lista de desafios que a humanidade enfrenta. A mudança climática é um dos maiores desafios do nosso tempo e seus efeitos negativos minam a capacidade de todos os países de alcançar o desenvolvimento sustentável. (p. 6)

Acrescente-se ao cenário a tendência de urbanização crescente da população mundial, que deve alcançar 70\% em 2050, com um aumento em dois bilhões de habitantes ${ }^{3}$. Diante de desafios tão contundentes, os países presentes comprometeram-se em angariar esforços transformadores nos quinze anos seguintes, tais como: acabar com a fome; combater as desigualdades; construir sociedades pacíficas, justas e inclusivas; proteger os direitos humanos e promover a igualdade de gênero; assegurar a proteção duradoura do planeta e seus recursos naturais.

A Agenda 2030 está organizada em 17 objetivos de desenvolvimento sustentável (ODS) e 169 metas associadas, com forte relação entre si e que devem ser analisadas de forma integrada e indissociável. Esta compreensão é coerente com a perspectiva ampliada e socialmente produzida de saúde consagrada no Brasil na lei orgânica de saúde (8.080/1990), de que saúde é socialmente produzida e se expressa de forma desigual em distintos grupos, dadas as suas inserções na sociedade. Os processos saúde-doença-cuidado e a distribuição de morbimortalidade num dado país ou região contém um conjunto de determinantes e condicionantes nos quais as desigualdades sociais correspondem às iniquidades em saúde ${ }^{4}$.

$\mathrm{O}$ atual contexto internacional coloca grandes barreiras para o avanço da Agenda 2030, dado o processo de fortalecimento do neoliberalismo e do neoconservadorismo em várias partes do mundo, que ameaçam os valores da solidariedade $^{5}$. Isso afeta o compromisso da humanidade com os direitos humanos e a superação de injustiças sociais, econômicas e ambientais, e abala diretamente os sistemas de proteção social, o que inclui as políticas nacionais de saúde que impactam diretamente o ODS 3 (Saúde e Bem-Estar).
Apesar da dimensão global desta Agenda, a estratégia política para a implementação dos ODS é de responsabilidade nacional ${ }^{2}$, cabendo ao governo de cada país determinar prioridades, estruturas de governança, monitoramento de resultados e formas de financiamento ${ }^{6}$.

Um dos destaques da Agenda é o ODS 2, dedicado ao tema "Fome Zero e Agricultura Sustentável”. Porém, desde a Resolução de 2015, quando o mundo se comprometeu a acabar com a fome, $a$ insegurança alimentar, melhorar a nutrição e promover a agricultura sustentável, os dados indicam que não há o que se comemorar, pelo contrário. O número de pessoas afetadas pela fome tem aumentado lentamente desde $2014^{7}$, no contexto de uma crise global de múltiplas dimensões: social, ética, econômica, democrática, ecológica e sanitária.

Há um amplo reconhecimento que, com a chegada e o avanço da pandemia de COVID-19, as desigualdades e as vulnerabilidades sociais preexistentes têm se agravado, afetando especialmente, além dos grupos de risco com comorbidades, certos países, territórios e populações. Destacam-se os mais pobres, negros, indígenas, trabalhadores precarizados, e ainda os profissionais de saúde que se encontram na linha de frente do atendimento à população. Nesse sentido, autores como Boaventura Santos ${ }^{8}$ aponta a atual crise como civilizatória, sendo a pandemia um marco que efetivamente inicia o tempo histórico do século XXI com seus desafios para a humanidade.

Existem relações intrínsecas entre o conjunto de metas do ODS 2 (Fome Zero e Agricultura Sustentável) e do ODS 3 (Saúde e Bem-Estar). Isso fica evidente nas conexões entre as metas de acabar com a fome (2.1) e todas as formas de desnutrição (2.2) com aquelas de reduzir a taxa de mortalidade materna global (3.1) e de acabar com as mortes evitáveis de recém-nascidos e crianças menores de 5 anos (3.2). O tema da alimentação possui especial transversalidade no conjunto dos ODS, sendo considerado fundamental para a saúde das pessoas e do planeta. Há razoável consenso de que grande parte dos problemas sociais e sanitários no mundo será resolvida somente na medida em que diversas ações articuladas em múltiplas escalas garantam alimentação saudável, acessível e de qualidade ao conjunto da população mundial.

O avanço da COVID-19 e as diversas crises que assolam o país vem reforçando o conceito de sindemia ${ }^{9}$, o qual se aproxima do de vulnerabilidade ${ }^{10}$, amplamente usado pelas ciências 
ambientais e da saúde desde os anos 1990. A vulnerabilidade é um conceito polissêmico desenvolvido por diversas disciplinas e campos de conhecimento voltados ao estudo de temas como desenvolvimento e sustentabilidade, pobreza e segurança alimentar e nutricional, desastres naturais e tecnológicos, mudanças climáticas globais e problemas de saúde pública, entre outros. Ele é utilizado para analisar porque certos países, regiões e grupos populacionais possuem consequências ou impactos mais graves frente a eventos de características similares como desastres e epidemias.

Já o conceito de sindemia amplia e complexifica os de epidemia e pandemia, ao mesmo tempo que complementa o de vulnerabilidade. Foi desenvolvido de forma interdisciplinar por epidemiologistas e antropólogos médicos nos EUA no diálogo entre a saúde, a economia política e a ecologia política. O conceito foi criado na década de $1990^{11}$ a partir de trabalhos pioneiros realizados com populações de periferias urbanas, moradores de rua e usuários de drogas. Posteriormente passou a ser usado num amplo espectro de problemas de saúde pública e saúde comunitária cujos efeitos das condições sociais interferem e agravam certas doenças combinadas, em particular as crônico degenerativas e transmissíveis como diabetes, hipertensão, obesidade e HIV, em associação com outros fatores de risco'.

O conceito de sindemia possui forte convergência com os princípios e bases conceituais da Saúde Coletiva, incluindo o diálogo com a teoria da determinação social. As pandemias relacionadas à fome, obesidade e mudanças climáticas foram considerados recentemente uma Sindemia $\mathrm{Global}^{12}$, pois interagem umas com as outras, compartilham de determinantes socioambientais comuns, e exercem uma influência mútua em sua carga de saúde para a sociedade.

Nesse sentido, a COVID-19 deve ser considerada uma nova sindemia que se soma às anteriores, sendo simultaneamente causa e consequência que se retroalimentam numa perspectiva complexa, tornando ainda mais difícil alcançar as metas negociadas globalmente pela Agenda 2030.

\section{Sistemas alimentares sustentáveis: conceitos-chave e documentos de referência}

Vem crescendo rapidamente o número de relatórios oficiais que pedem mudanças fundamentais nos sistemas alimentares para torná-los mais saudáveis, sustentáveis e equitativos ${ }^{12}$. Com diferentes abordagens, tais documentos têm ana- lisado as relações complexas entre alimentação, saúde, meio ambiente e agricultura, demonstrando a interdependência dos objetivos da Agenda 2030, em especial os ODS 2 e 3.

As resoluções aprovadas pela Assembleia Geral da ONU que proclamaram as Década de Ação pela Nutrição (2016-2025), Década da Agricultura Familiar (2019-2028) e Década para a Restauração do Ecossistema (2021-2030), bem como a declaração das nações unidas sobre os direitos dos camponeses e de outras pessoas que trabalham em áreas rurais (2018), reforçam e estimulam esforços para a transformação de sistemas alimentares, ao mesmo tempo que indicam que relatórios internacionais seguirão se acumulando sobre o tema nos próximos anos.

Temas tão complexos exigem uma abordagem sistêmica que incorpore conceitos estratégicos desenvolvidos nas últimas décadas, e no artigo destacamos quatro deles. O primeiro é o de sistema alimentar, compreendido como o conjunto de elementos (ambiente, pessoas, insumos, processos, infraestrutura, instituições e organizações da sociedade civil, dentre outros) e atividades que se interrelacionam na produção, processamento, distribuição, preparação e consumo de alimentos, o que inclui as características e os resultados dos sistemas socioeconômicos e ambientais dessas atividades ${ }^{13}$. Muitos autores consideram mais apropriado o termo Sistemas Agroalimentares. Neste ensaio trataremos os termos como sinônimos e adotaremos sistemas alimentares, que é a forma como tem sido mais adotado em relatórios internacionais, documentos de suporte para este texto.

Um sistema alimentar pode ser mais ou menos complexo a depender da distância entre produtores e consumidores, bem como o número de passagens por intermediários diversos, incluindo comerciantes, industriais e transportadores. São constituídos por circuitos alimentares diversos e superpostos, os quais podem formar desde um modelo de comunidades camponesas vivendo em autoconsumo em agricultura de subsistência, passando por circuitos de mercados locais, regionais e/ou nacionais, com transformação dos alimentos ou não. Em escalas maiores podem ser formados circuitos de economias planificadas (com maior intervenção do Estado) e/ou circuito internacional, caso típico do comércio de commodities característico do agronegócio de exportação, o qual constitui o modelo hegemônico no Brasil em termos de poder econômico e político. Em uma mesma região ou país vários circuitos podem funcionar ao mesmo tempo, a depender 
dos produtos alimentícios, das relações entre populações rurais e urbanas, da penetração de grandes empresas e indústrias, das características econômicas mais relacionadas ao mercado externo ou interno, ou ainda de aspectos ecológicos e culturais que conformam a complexidade dos circuitos alimentares em diferentes sociedades e agroecossistemas $^{14}$.

Considera-se um sistema alimentar sustentável (SAS) aquele que cumpre sua função social, isto é, que proporciona Segurança Alimentar e Nutricional (SAN) para todas as pessoas, sem comprometer as bases econômicas, sociais e ambientais que geram segurança alimentar e nutricional para as gerações futuras ${ }^{13}$.

$\mathrm{O}$ conceito de SAN é o segundo estratégico por nós adotado. Consagrado no Brasil desde 2006 no final do primeiro governo Lula como um dos marcos do seu Programa Fome Zero e consiste na realização do direito de todos ao acesso regular e permanente a alimentos de qualidade, em quantidade suficiente, sem comprometer o acesso a outras necessidades essenciais. Tem como base práticas alimentares promotoras de saúde que respeitem a diversidade cultural e que sejam ambiental, cultural, econômica e socialmente sustentáveis (Art. 3$)^{15}$.

Esse referencial foi construído com base na participação social, formulação de políticas públicas e pesquisas acadêmicas que passam pela recriação do Conselho Nacional de Segurança Alimentar e Nutricional (Consea) e desenvolvimento do programa Fome Zero, em 2003, e um conjunto de políticas públicas correlatas, incluindo a realização da II Conferência Nacional de Segurança Alimentar e Nutricional (2004). Todos esses processos sedimentaram as noções de soberania alimentar e de garantia ao direito humano à alimentação adequada e saudável, fundamentais para a organização de sistemas alimentares justos e sustentáveis ${ }^{16}$.

O terceiro conceito estratégico é o de agroecologia, que tem ganhado reconhecimento global a partir de um conjunto significativo de evidências científicas e empíricas que contribuem para tornar efetivo o direito humano à alimentação adequada. Este último completa o conjunto dos quatro conceitos estratégicos e é caracterizado a partir de suas cinco dimensões: disponibilidade, acessibilidade, adequação, sustentabilidade e participação ${ }^{17}$. A realização de dois Simpósios Internacionais sobre Agroecologia para a SAN, organizados pela FAO, em 2014 e 2018, bem como a III Conferência Internacional Agricultura e Alimentação em uma Sociedade Urbanizada
(2018) realizada no Brasil, possuem no centro das reflexões a necessidade de mudanças no sistema alimentar global e de aumentar a escala da agroecologia para atingir $\mathrm{ODS}^{18}$.

A agroecologia pode ser compreendida como uma forma de redesenhar sistemas alimentares, desde o estudo e manejo de agroecossistemas rurais ou urbanos até a mesa dos consumidores, com o objetivo de alcançar sustentabilidade com preservação ambiental, viabilidade econômica e justiça social. Por meio de pesquisas e ações orientadas para a mudança de natureza transdisciplinar, intercultural e participativa, a agroecologia une ciência, práticas agrícolas, movimentos da sociedade civil e políticas públicas focadas na transformação social ${ }^{19,20}$, sendo estratégica no redesenho e transição dos sistemas e circuitos alimentares.

Selecionamos nesse artigo dez relatórios internacionais publicados entre 2016 e 2020, que contribuem com abordagens sobre sistemas alimentares e possibilitam um diálogo transversal entre o ODS 2 da Agenda 2030 e a saúde. São dois documentos de cada um dos três grupos internacionais de especialistas em SAN: (1) Painel de Especialistas em SAN (HLPE) do Comitê de Segurança Alimentar Mundial/ONU; (2) Painel Internacional de Especialistas em Sistemas Alimentares Sustentáveis (IPES-Food); (3) Comissão EAT-Lancet sobre Alimentos, Planeta, Saúde. Nos casos do HLPE e do IPES-Food, em que há mais relatórios publicados no período, foram priorizados aqueles que trazem abordagens mais amplas de sistemas alimentares. Adicionamos à seleção relatórios oficiais que contribuem com um panorama geral sobre sistemas alimentares, seja com focos mais em SAN e impactos de dietas: a versão de 2020 de "O estado da Segurança Alimentar e Nutricional no Mundo" e a de 2019 do "Situação Mundial da Infância", que tem como tema "Crianças, Alimento e Nutrição". Por fim, foram incluídos dois documentos que contribuem para a compreensão complexa e profunda das relações entre sistemas alimentares e sustentabilidade ambiental: um sobre mudanças climáticas, outro sobre biodiversidade e serviços ecossistêmicos (Quadro 1).

\section{A importância de sistemas alimentares para a Saúde Global e a Agenda 2030}

Entre 1990 e meados da segunda década do século XXI houve avanços significativos na diminuição da proporção mundial de adultos e crianças que sofrem de algum grau de desnutrição. Ses- 
Quadro 1. Relatórios internacionais selecionados sobre Sistemas Alimentares, por ano de publicação.

\begin{tabular}{|c|c|c|}
\hline Autoria & Título & Ano \\
\hline IPES-Food & $\begin{array}{l}\text { Da Uniformidade à Diversidade } \\
\text { Mudança de paradigma: da agricultura industrial para sistemas } \\
\text { agroecológicos diversificados }\end{array}$ & 2016 \\
\hline IPES-Food & $\begin{array}{l}\text { Desvendando a relação alimento-saúde } \\
\text { Abordando práticas, economia política e relações de poder para } \\
\text { construir sistemas alimentares saudáveis }\end{array}$ & 2017 \\
\hline HLPE & A nutrição e os sistemas alimentares & 2018 \\
\hline $\begin{array}{l}\text { Comissão EAT-Lancet sobre } \\
\text { Alimentos, Planeta, Saúde }\end{array}$ & $\begin{array}{l}\text { A sindemia global da obesidade, desnutrição e mudanças } \\
\text { climáticas }\end{array}$ & 2019 \\
\hline $\begin{array}{l}\text { Comissão EAT-Lancet sobre } \\
\text { Alimentos, Planeta, Saúde }\end{array}$ & $\begin{array}{l}\text { Alimentos no Antropoceno: a Comissão EAT-Lancet sobre dietas } \\
\text { saudáveis a partir de sistemas alimentares sustentáveis }\end{array}$ & 2019 \\
\hline HLPE & $\begin{array}{l}\text { Enfoques agroecológicos e outros enfoques inovadores em favor } \\
\text { da sustentabilidade da agricultura e os sistemas alimentares que } \\
\text { melhoram a segurança alimentar e nutriciona. }\end{array}$ & 2019 \\
\hline $\begin{array}{l}\text { Fundo das Nações Unidas para } \\
\text { a Infância (UNICEF) }\end{array}$ & $\begin{array}{l}\text { Situação Mundial da Infância } 2019 \\
\text { Crianças, alimentação e nutrição - Crescendo saudável em um } \\
\text { mundo em transformação }\end{array}$ & 2019 \\
\hline $\begin{array}{l}\text { Plataforma Intergovernamental } \\
\text { de Política Científica sobre } \\
\text { Biodiversidade e Serviços } \\
\text { Ecossistêmicos (IPBES) }\end{array}$ & $\begin{array}{l}\text { Relatório de avaliação global sobre biodiversidade e serviços } \\
\text { ecossistêmicos }\end{array}$ & 2019 \\
\hline $\begin{array}{l}\text { FAO, OMS, FIDA, PMA e } \\
\text { UNICEF** }^{*}\end{array}$ & $\begin{array}{l}\text { O estado da segurança alimentar e nutricional no mundo. } \\
\text { Transformação dos sistemas alimentares para que promovam } \\
\text { dietas acessiveis e saudáveis }\end{array}$ & 2020 \\
\hline $\begin{array}{l}\text { Grupo Intergovernamental de } \\
\text { Especialistas sobre o Cambio } \\
\text { Climático (IPCC) }\end{array}$ & $\begin{array}{l}\text { A mudança climática e a terra } \\
\text { Relatório especial do IPCC sobre mudanças climáticas, } \\
\text { desertificação, degradação da terra, gestão sustentável da } \\
\text { terra, segurança alimentar e fluxos de gases de efeito estufa em } \\
\text { ecossistemas terrestres }\end{array}$ & 2020 \\
\hline
\end{tabular}

* os títulos dos relatórios / documentos desse quadro foram traduzidos de forma livre pelos autores. ${ }^{\star *}$ FAO - Organização das Nações Unidas para a Agricultura e a Alimentação; OMS - Organização Mundial da Saúde; FIDA - Fundo Internacional de Desenvolvimento Agrícola; PMA - Programa Mundial de Alimentos.

Fonte: Elaborado pelos autores.

senta países considerados em desenvolvimento cumpriram ou superaram as metas dos Objetivos de Desenvolvimento do Milênio (2000-2015) de reduzir pela metade a proporção de pessoas que sofrem de fome ${ }^{21}$. Porém, o número de pessoas afetadas por insegurança alimentar grave, em nível mundial, vem aumentando lentamente desde 2014, atingindo cerca de 750 milhões de pessoas em $2019^{7}$. Estimativas para 2015 indicavam que cerca de dois bilhões de pessoas eram afetadas pela deficiência de micronutrientes (DMNs ou "fome oculta") e outros quase dois bilhões por sobrepeso ou obesidade ${ }^{22}$. Há pelo menos quatro décadas a pandemia de obesidade vem mudando o padrão de má nutrição e atualmente avança em todas as regiões do mundo em ritmo constante, afetando países de alta, média e baixa renda ${ }^{7,12}$.
A má nutrição em todas as suas formas, incluindo a desnutrição, a obesidade e outros riscos alimentares para doenças crônicas não transmissíveis (DCNTs) representa atualmente a principal causa de doenças e mortes prematuras no mundo todo $^{12,23}$. A Comissão The Lancet destaca que a obesidade e seus determinantes guardam relação com três das quatro principais causas de DCNTs no planeta, incluindo doenças cardiovasculares, diabetes tipo 2 e certos tipos de câncer (p. 17), e que os custos econômicos anuais globais da obesidade são de aproximadamente US\$ 2 trilhões $(2,8 \% \text { do PIB mundial })^{12}$.

As crianças e mulheres em gestação são particularmente vulneráveis a tripla carga da má nutrição - desnutrição, DMNs e sobrepeso - que ameaça a sobrevivência e prejudica a capacidade 
de milhões de crianças e adolescentes de crescer e desenvolver todo o seu potencial, impacta de forma relevante a saúde materna, com consequências imediatas e de médio e longo prazos (Quadro 2). Pelo menos uma em cada três crianças menores de 5 anos no mundo está desnutrida ou com sobrepeso, e uma em cada duas sofre de fome oculta. Esse cenário perpetua a pobreza entre as gerações e as regiões, tendo em vista que a maior carga de todas as formas de má nutrição em crianças e adolescentes se concentra nas comunidades mais pobres e marginalizadas ${ }^{23}$.

Os relatórios analisados apontam que os sistemas alimentares globais na atualidade são incapazes de oferecer dietas saudáveis ${ }^{12,21-25}$. Trata-se de um sistema hegemonizado pela agricultura capitalista global que conforma os impérios alimentares, formado pelo agronegócio produtor de commodities, pelas corporações transnacionais industriais e logísticas responsáveis pelos insumos, beneficiamento, transporte e comercialização, incluindo as cadeias de supermercados, bem como o setor financeiro ${ }^{26}$.

O controle sobre a produção, processamento e comercialização de alimentos, assim como estratégias de marketing, possibilitou as corporações a induzirem o consumo de alimentos industrializados e processados que são ricos em gorduras trans, açúcar, sal e aditivos químicos, que cresce na maioria dos países e corresponde a principal razão para o aumento da prevalência de sobrepeso e obesidade. A grande escala da produção e os benefícios fiscais obtidos em vários países tendem a tornar mais baratos tais alimentos em relação aos alimentos nutritivos e frescos ${ }^{12}$. Estimou-se que mais de três bilhões de pessoas no mundo não possuem recursos para pagar por dietas saudáveis, que custam, em média, cinco vezes mais que as dietas que só atendem as necessidades energéticas por meio de alimentos ricos em amido e pobres em nutrientes?

Os sistemas alimentares globais são também considerados a principal causa de mudança ambiental no planeta. A expansão das áreas agrícolas, de pecuária e de florestais plantadas nas últimas décadas contribuíram para o desmatamento e aumentar as emissões líquidas de Gases de Efeito Estufa (GEE), com a degradação de ecossistemas naturais (como florestas, savanas, pastagens naturais e pântanos) e declínio da biodiversidade. Atividades de agricultura, silvicultura e outros usos da terra representam $16-27 \%$ das emissões de GEE (ou de $21-37 \%$ se considerarmos atividades de pré e pós-produção do sistema alimentar), sendo que a pecuária em pastagens foi responsável por mais da metade das emissões antropogênicas totais de $\mathrm{N}_{2} \mathrm{O}$ em $2014^{27}$.

O IPCC considera que a mudança climática já afetou a SAN devido ao aquecimento, mudanças nos padrões de precipitação e maior frequên-

Quadro 2. Como a tripla carga de má nutrição prejudica crianças, adolescentes e mulheres.

\begin{tabular}{|l|l|}
\hline \multicolumn{2}{|c|}{ Crianças e adolescentes } \\
\hline $\begin{array}{l}\text { Subnutrição, desnutrição crônica } \\
\text { (baixa estatura para a idade) e } \\
\text { desnutrição aguda } \\
\text { (baixo peso para a altura) }\end{array}$ & $\begin{array}{l}\text {. Baixo crescimento, infecção e morte } \\
\text {. Baixo desenvolvimento cognitivo, falta de atenção escolar e desempenho } \\
\text { escolar fraco } \\
\text {. Baixo potencial de ganho financeiro na vida adulta }\end{array}$ \\
\hline $\begin{array}{l}\text { Fome oculta - deficiências em } \\
\text { micronutrientes }\end{array}$ & $\begin{array}{l}\text {. Baixo crescimento e desenvolvimento } \\
\text {. Imunidade fraca e fraco desenvolvimento de tecidos } \\
\text {. Saúde precária e risco de morte }\end{array}$ \\
\hline Sobrepeso (incluindo obesidade) & $\begin{array}{l}\text {. A curto prazo: problemas cardiovasculares, infecções e baixa autoestima } \\
\text {. A longo prazo: obesidade, diabetes e outros distúrbios metabólicos }\end{array}$ \\
\hline $\begin{array}{l}\text { Subnutrição, déficit de } \\
\text { crescimento e baixo peso }\end{array}$ & $\begin{array}{l}\text {. Mulheres grávidas } \\
\text {. Parto prematuro e baixo peso ao nascer } \\
\text {. Doenças crônicas para a criança, ao longo da vida }\end{array}$ \\
\hline $\begin{array}{l}\text { Fome oculta - deficiências em } \\
\text { micronutrientes }\end{array}$ & $\begin{array}{l}\text {. Mortalidade e morbidade materna } \\
\text {. Defeitos no tubo neural em recém-nascidos } \\
\text {. Parto prematuro, baixo peso ao nascer e comprometimento do } \\
\text { desenvolvimento cognitivo em recém-nascidos }\end{array}$ \\
\hline $\begin{array}{l}\text { Sobrepeso (incluindo obesidade) } \\
\text {. Diabetes gestacional e pré-eclâmpsia } \\
\text {. Complicações obstétricas } \\
\text {. Excesso de peso e doença crônica nas crianças ao longo da vida }\end{array}$ \\
\hline
\end{tabular}

Fonte: UNICEF, 2019. 
cia de alguns eventos extremos. A produção de frutas e legumes - elemento chave de dietas saudáveis - é particularmente vulnerável a mudanças climáticas. Extremos climáticos têm impactos sobre meios de subsistência de comunidades mais pobres e vulneráveis, contribuindo para as migrações. Diversas pesquisas indicam que níveis aumentados de dióxido de carbono na atmosfera estão reduzindo os níveis de nutrientes em alimentos ${ }^{12}$.

A perda de biodiversidade é o limite planetário mais impactado pela ação humana, e estimase que isso poderá prejudicar o progresso em até $80 \%$ das metas avaliadas dos ODS relacionadas à pobreza, alimentação, saúde, água, cidades, clima, oceanos e terra. A produção de alimentos pode ser comprometida pela perda de polinizadores naturais, pois $75 \%$ dos tipos de culturas alimentares globais contam com a polinização animal, mas os métodos agrícolas intensivos agrotóxicodependentes ameaçam extinguir esses tais serviços ecossistêmicos ${ }^{28}$.

Considera-se que toda dieta tem custos ocultos e que guardam sinergias por suas consequências com a saúde (ODS 3) e com o clima (ODS 13), a depender dos alimentos consumidos e os sistemas alimentares dos quais fazem parte ${ }^{7}$.

Uma abordagem a partir dos sistemas alimentares permite identificar cinco canais-chave que influenciam a saúde: 1) riscos ocupacionais; 2) contaminação ambiental; 3) alimentos contaminados, inseguros e alterados; 4) padrões alimentares não saudáveis; e, 5) insegurança alimentar. O sistema alimentar global hegemônico contribui decisivamente para tais canais, provocando prejuízos humanos e econômicos que ameaçam o desenvolvimento da humanidade e a saúde do planeta. A contaminação do solo, ar e águas com fertilizantes, agrotóxicos e antibióticos, por exemplo, expõe diferentes seres vivos a inúmeras consequências. Estima-se que somente nos EUA, por ano, a resistência a antimicrobianos tenha gerado oito milhões de dias adicionais de internação e custos à saúde entre US\$ 20 a 34 bilhões ${ }^{21}$.

Os diferentes relatórios considerados neste artigo convergem para um consenso de que mudanças profundas e transformadoras no sistema alimentar global são necessárias e urgentes ${ }^{12}$. Os esforços dos diferentes grupos em analisar os dados de forma integrada e propor caminhos de transição reúne acúmulos bastante significativos.

A Comissão EAT-Lancet defende um pacto de reorganização do sistema alimentar global com o compromisso de avançar para dietas que promovam saúde planetária para quase dez bilhões de pessoas em 2050. A proposta envolve mais que dobrar o consumo de alimentos saudáveis, como frutas, vegetais, legumes e nozes, e uma redução de mais de $50 \%$ no consumo global de alimentos menos saudáveis, como açúcares adicionados e carne vermelha (p. 12), além de reduzir pela metade o desperdício de alimentos no mundo e conter para que a área destinada a produção de alimentos não avance ${ }^{24}$. A UNICEF reivindica que as necessidades nutricionais das crianças devem estar no centro dos sistemas alimentares nacionais como condição fundamental para o desenvolvimento sustentável ${ }^{23}$. Contudo, para isso será necessário transformar não apenas as bases argumentativas, mas as políticas públicas, econômicas e simbólicas dominantes que sustentam o atual regime alimentar global ${ }^{29}$.

O reconhecimento internacional crescente da importância da agroecologia para a promoção de sistemas alimentares sustentáveis se expressa de forma heterogênea em alguns dos relatórios. Curiosamente, a Comissão EAT-Lancet sequer cita agroecologia em seus estudos. O IPCC e o IPBES destacam em seus documentos as contribuições da agroecologia na promoção da SAN desde o manejo de agroecossistemas à reorganização de sistemas alimentares que possibilitam a mitigação das mudanças climáticas e a preservação da biodiversidade através de sistemas mais diversificados e resilientes ${ }^{26,27}$.

O painel de especialistas em SAN vinculado a FAO (HLPE) defende um enfoque agroecológico de sistemas alimentares sustentáveis para promoção do direito humano à alimentação adequada ${ }^{30}$. O HLPE apresenta 13 princípios da abordagem agroecológica (Quadro 3) em diálogo com compilados anteriores, entre eles "Os 10 elementos da Agroecologia”"31 , com uma visão holística que coloca a agroecologia como alternativa central para o futuro do planeta e da humanidade.

As abordagens agroecológicas favorecem o uso de processos naturais, limitam o uso de insumos adquiridos, promovem ciclos fechados com externalidades negativas mínimas e enfatizam a importância do conhecimento local e processos participativos que desenvolvem conhecimentos e práticas através da experiência, bem como métodos científicos mais convencionais, e enfrentam as desigualdades sociais. As abordagens agroecológicas reconhecem que os sistemas agroalimentares são sistemas sociais e ecológicos combinados que vão da produção ao consumo de alimentos e envolvem a participação da ciência, da prática e de um movimento social, bem como sua integração holística, para abordar a segurança alimentar e a nutrição. (p. 43, tradução nossa) 
Quadro 3. Princípios agroecológicos, desde compilação do HLPE.

\begin{tabular}{|c|c|c|}
\hline Princípio & $\begin{array}{c}\text { Os } 10 \text { elementos da } \\
\text { Agroecologia (FAO) }\end{array}$ & $\begin{array}{c}\text { Escala de } \\
\text { aplicação* }^{*}\end{array}$ \\
\hline \multicolumn{3}{|c|}{ Melhorar a eficiência na utilização de recursos } \\
\hline $\begin{array}{l}\text { 1. Reciclagem. Utilizar preferivelmente recursos locais renováveis e, na } \\
\text { medida do possível, fechar os ciclos de recursos de nutrientes e biomassa }\end{array}$ & Reciclagem & CA, AA \\
\hline $\begin{array}{l}\text { 2. Redução de insumos. Reduzir ou eliminar a dependência de insumos } \\
\text { comprados e aumentar a autossuficiência }\end{array}$ & Eficiência & AA, SA \\
\hline \multicolumn{3}{|l|}{ Fortalecer a resiliência } \\
\hline $\begin{array}{l}\text { 3. Saúde dos solos. Garantir a saúde e o funcionamento dos solos - e } \\
\text { fortalecê-los - para melhorar o crescimento das plantas em particular, } \\
\text { fazendo a gestão da matéria orgânica e reforçando a atividade biológica do } \\
\text { solo }\end{array}$ & $\begin{array}{l}\text { Diversidade; } \\
\text { Resiliência }\end{array}$ & $\mathrm{CA}$ \\
\hline 4. Saúde animal. Garantir a saúde e o bem estar dos animais & Resiliência & $\mathrm{CA}, \mathrm{AA}$ \\
\hline $\begin{array}{l}\text { 5. Biodiversidade. Manter e melhorar a diversidade de espécies, diversidade } \\
\text { funcional e recursos genéticos e, assim, manter a biodiversidade geral do } \\
\text { agroecossistema no tempo e no espaço, no campo, nas atividades agrícolas } \\
\text { e no território }\end{array}$ & Diversidade & $\mathrm{CA}, \mathrm{AA}$ \\
\hline $\begin{array}{l}\text { 6. Sinergias. Melhorar a interação ecológica positiva, a sinergia, a integração } \\
\text { e a complementaridade entre os elementos dos agroecossistemas (animais, } \\
\text { cultivos, árvores, solo e água) }\end{array}$ & Sinergia & CA, AA \\
\hline $\begin{array}{l}\text { 7. Diversificação econômica. Diversificar a renda agrícola, garantindo } \\
\text { que os pequenos agricultores tenham maior independência financeira } \\
\text { e possibilidades de agregar valor, ao mesmo tempo que lhes permite } \\
\text { responder à demanda de consumidores }\end{array}$ & Diversidade & AA, SA \\
\hline \multicolumn{3}{|l|}{ Garantir equidade e responsabilidade social } \\
\hline $\begin{array}{l}\text { 8. Criação conjunta de conhecimentos. Melhorar a cocriação e o intercâmbio } \\
\text { horizontal de conhecimento, incluindo inovação local e científica, } \\
\text { especialmente por meio do intercâmbio entre agricultores }\end{array}$ & $\begin{array}{l}\text { Criação conjunta } \\
\text { e intercâmbio de } \\
\text { conhecimentos }\end{array}$ & AA, SA \\
\hline $\begin{array}{l}\text { 9. Valores sociais e dietas. Construir sistemas alimentares baseados } \\
\text { na cultura, identidade, tradição e equidade social e de gênero das } \\
\text { comunidades locais, que proporcionem dietas saudáveis, diversificadas e } \\
\text { adequadas do ponto de vista sazonal e cultural }\end{array}$ & $\begin{array}{l}\text { Valores humanos } \\
\text { e sociais; Cultura } \\
\text { e tradições } \\
\text { alimentares }\end{array}$ & AA, SA \\
\hline $\begin{array}{l}\text { 10. Imparcialidade. Apoiar meios de subsistência decentes e sólidos para } \\
\text { todos os atores envolvidos nos sistemas alimentares, especialmente os } \\
\text { pequenos produtores de alimentos, com base no comércio justo, no } \\
\text { emprego equitativo e no tratamento imparcial dos direitos de propriedade } \\
\text { intelectual }\end{array}$ & Resiliência & AA, SA \\
\hline $\begin{array}{l}\text { 11. Conectividade. Garantir a proximidade e a confiança entre produtores e } \\
\text { consumidores através da promoção de redes de distribuição justas e curtas } \\
\text { e da reincorporação dos sistemas alimentares nas economias locais }\end{array}$ & $\begin{array}{l}\text { Economia circular e } \\
\text { solidária }\end{array}$ & AA \\
\hline $\begin{array}{l}\text { 12. Governança da terra e dos recursos naturais. Fortalecer os arranjos } \\
\text { institucionais para melhorar o reconhecimento e o apoio aos agricultores } \\
\text { familiares, camponeses e pequenos produtores de alimentos como gestores } \\
\text { sustentáveis dos recursos naturais e genéticos }\end{array}$ & $\begin{array}{l}\text { Governança } \\
\text { responsável }\end{array}$ & AA, SA \\
\hline $\begin{array}{l}\text { 13. Participação. Promover a organização social e uma maior participação } \\
\text { de produtores e consumidores no processo de tomada de decisão, para } \\
\text { apoiar a governança descentralizada e a gestão de sistemas agrícolas e } \\
\text { alimentares adaptados às condições locais }\end{array}$ & $\begin{array}{l}\text { Governança } \\
\text { responsável; } \\
\text { Resiliência }\end{array}$ & SA \\
\hline
\end{tabular}

${ }^{*}$ Escala de aplicação: $\mathrm{CA}=$ campo; $\mathrm{AA}=$ atividade agropecuária, agroecossistema; $\mathrm{SA}=$ sistema alimentar

Fonte: Traduzido e adaptado de HLPE, 2019.

O IPES-Food atua com foco nos elementos que propiciam avanços da transição para "sistemas alimentares agroecológicos” (SAA), disponí- veis em um conjunto de publicações. Em estudo comparado ao sistema alimentar global ${ }^{22}$, ficam evidentes como os SAA produzem respostas a 
um conjunto de problemas interligados, com o potencial de contribuir para o avanço de várias das metas dos ODS.

Destacamos duas necessidades fundamentais para as mudanças almejadas: 1) defender e fortalecer a integridade da pesquisa científica como um bem público ${ }^{21}$, livre de conflitos de interesses com setores econômicos interessados em manter suas taxas de lucro a despeito dos impactos sociais, ambientais e sanitários. Esforços interdisciplinares, incluindo a Saúde Coletiva, são necessários para melhor compreender e impulsionar os sistemas alimentares sustentáveis e a agroecologia; 2) é necessário enfrentar as desigualdades de poder dentro dos sistemas alimentares em todos os níveis e diferentes dimensões, o que inclui proteger países e grupos populacionais vulneráveis a práticas exploratórias e predatórias de empresas de alimentos e bebidas ${ }^{25}$. Isso implica a participação da sociedade civil organizada em processos de governança horizontalizados. Deve-se mobilizar e garantir o protagonismo de mulheres, jovens, consumidores, entre outros.

\section{Considerações finais}

Dietas de baixa qualidade nutricional são o principal fator de risco para a carga global de doenças e estão intrinsecamente relacionadas com impactos antrópicos nas mudanças climáticas e perda de biodiversidade aceleradas nas últimas décadas. Os grupos populacionais mais pobres e vulneráveis sofrem as maiores cargas desses impactos, condenando-os à pobreza ao mesmo tempo que grupos poderosos concentram riqueza. Os sistemas alimentares globais, resultados do neoliberalismo, da expansão da agricultura industrial capitalista e dos impérios alimentares ${ }^{26}$, contribuem decisivamente para esse cenário. Trata-se, portanto, de um tema estratégico para a saúde global nas próximas décadas ou mesmo séculos.

A origem do conceito de sistemas alimentares é localizada no contexto da crise alimentar mundial de 1972, que se desdobrou na "Conferência Mundial de Alimentos" de 1974 e na "Declaração Universal para Eliminação Definitiva da Fome e da Desnutrição"14. Após cinco décadas o problema não foi resolvido, em que pese a atuação de vários países: existe uma sindemia global de desnutrição, obesidade e mudanças climáticas. Para piorar, o avanço da pandemia de COVID-19 trouxe grandes repercussões para a saúde da humanidade, incluindo o agravamento de desigualdades e vulnerabilidades sociais preexistentes.
Estima-se que, dentre os efeitos negativos desta pandemia, o número de pessoas em situação de fome no mundo em 2020 tenha aumentado entre 83 a 132 milhões ${ }^{7}$.

Por suas dimensões continentais, produção agropecuária, concentração de riquezas naturais e ampla diversidade sociocultural, o Brasil tem se destacado nos debates sobre sistemas alimentares, porém nos últimos anos tem havido retrocessos importantes. A reprimarização da economia brasileira nas últimas décadas, apoiada pelo Estado, se expressou no boom de commodities, com a expansão do gado de corte e os cultivos de soja, milho e cana-de-açúcar, especialmente. Tais monocultivos ameaçam ainda mais biomas ricos em sociobiodiversidade e vitais para os equilíbrios hídrico e climático, como a Amazônia e o Cerrado. O Censo Agropecuário de 2017 revelou, em comparação com o de 2006, as consequências negativas do avanço do agronegócio, cujos agentes econômicos lucram bilhões de reais por ano: a concentração fundiária e de renda aumentou, com redução de $9,5 \%$ dos estabelecimentos agropecuários da agricultura familiar, redução em postos de trabalho e pessoal ocupado, aumento do uso de agrotóxicos, e diminuição da produção de alimentos diversificados ${ }^{32}$. Do outro lado da cadeia de alimentos, os grandes supermercados concentram cada vez mais poder e renda, reforçando o consumo de alimentos ultraprocessados e contaminados ${ }^{33}$ em uma sociedade cada vez mais urbanizada. Ao mesmo tempo, agricultores familiares fornecedores de alimentos mais saudáveis deixam de ser apoiados.

$\mathrm{O}$ até recentemente ministro do meio ambiente do Brasil defendeu que a atenção concentrada na COVID-19 seja aproveitada para mudanças (infra)legais que orientam a atuação do Estado brasileiro. O termo "passada da boiada" se reflete no desmonte da proteção ambiental, nos recordes de liberação de agrotóxicos e no desmatamento e queimadas que marcam o Governo Bolsonaro. Este, no primeiro dia de mandato, extinguiu o Conselho Nacional de Segurança Alimentar e Nutricional, além de ter fragilizado um conjunto de políticas de SAN da qual o país já foi referência internacional ${ }^{34}$. Enquanto o agronegócio comemora seus ganhos econômicos, a insegurança alimentar avança de forma acelerada no país ${ }^{35}$.

No contexto da Década de Ações para o alcance dos ODS até 2030, a ONU realizará em 2021 a Cúpula Mundial de Sistemas Alimentares. Relatores Especiais da ONU para o Direito à Alimentação denunciaram a apropriação da condu- 
ção da Cúpula por atores articulados ao Fórum Econômico Mundial com um esvaziamento do lugar central do Comitê de Segurança Alimentar Mundial da ONU enquanto espaço de governança dos sistemas alimentares globais. Com a participação da sociedade civil prejudicada, a Cúpula tem sua legitimidade comprometida ${ }^{36}$. Se, por um lado, a Agenda 2030 representa um pacto em torno dos direitos humanos e da saúde do planeta, de outro o poder econômico concentrado em grandes corporações atua para obstaculizar o avanço das mudanças pactuadas, sendo este um dos impasses centrais.

Mas existem esperanças: a agroecologia representa um paradigma emergente que continua a avançar no século XXI, sendo o movimento agroecológico brasileiro uma referência mundial de mudança ${ }^{22}$. Destacamos os resultados de duas pesquisas realizadas em 2020 para ilustrar a riqueza dos esforços para a construção de sistemas alimentares locais, diversos, sustentáveis e saudáveis com participação decisiva do campo agroecológico.

O projeto "Ação Coletiva Comida de Verdade: aprendizagem em tempos de pandemia" mapeou 310 iniciativas criadas ou adaptadas e adensadas durante os primeiros meses da pandemia no país. Protagonizadas por organizações populares, $\mathrm{co}-$ letivos, redes e movimentos sociais de todo o país, do campo e da cidade, essas experiências revelam a resiliência e a diversidade de sistemas alimentares locais comprometidos em garantir comida de verdade $^{37}$. A pesquisa "Municípios Agroecológicos e Políticas de Futuro - iniciativas municipais de apoio à agricultura familiar e à agroecologia $e$ de promoção da SAN" identificou 721 iniciativas, em 531 municípios de 26 estados, que apoiam, direta ou indiretamente, a agroecologia. A diversidade dessas experiências envolve 41 temas ${ }^{38}$ que reforçam a coerência de pensar a agroecologia como ecologia de sistemas alimentares ${ }^{19}$.
A análise dessas experiências revela estratégias, desafios, perspectivas, limites e avanços. $\mathrm{O}$ trabalho em rede na condução dessas pesquisas comprometidas com a ação segue em curso, buscando promover interação entre essas experiências para fortalecimento e criação de novas iniciativas e formas de produção de conhecimento.

Alimentar a humanidade com dietas nutritivas, garantir o direito ao meio ambiente equilibrado, reduzir as desigualdades e injustiças sociais, sanitárias e ambientais, promover a saúde e o bem estar são metas complementares e interdependentes que podem ser promovidas por meio de sistemas alimentares sustentáveis. Defende-se que os enfoques de segurança alimentar e nutricional, direito humano à alimentação adequada e agroecologia são fundamentais para a redemocratização e a sustentabilidade dos sistemas alimentares. Trata-se de uma abordagem orgânica para um conjunto de problemas e desafios que impactam a saúde global.

Neste artigo, defendemos a importância dos sistemas alimentares para a saúde e a Agenda 2030, no contexto de uma sindemia que já estava em curso e se retroalimenta com a COVID-19. Reconhecemos os limites desse artigo em tratar do conjunto de questões abordadas em relatórios tão complexos e complementares. Há outros relatórios disponíveis dos mesmos grupos e organizações selecionados que complementam e ampliam contribuições críticas sobre os sistemas alimentares, bem como recomendações para mudanças. Questões como desperdício de alimentos, contaminação dos oceanos, exploração dos alimentos do mar e a importância da pesca artesanal são exemplos de lacunas desse texto. Defendemos uma atenção mais estratégica do campo da saúde coletiva para os sistemas alimentares, aproximando mais e mais seu envolvimento com o campo agroecológico no Brasil, intensificando suas contribuições em prol das transformações necessárias para a saúde do planeta ${ }^{18}$.

\section{Colaboradores}

AC Burigo participou de todas as etapas do artigo, e MFS Porto participou da análise e interpretação dos dados, revisão e aprovação da versão final do texto. 


\section{Agradecimento}

Agradecemos o apoio do Programa de Pós-Graduação em Saúde Pública da Escola Nacional de Saúde Pública Sergio Arouca, da Fundação Oswaldo Cruz (ENSP/Fiocruz).

\section{Referências}

1. Fortes PAC, Ribeiro H. Saúde Global em tempos de globalização. Saúde e Sociedade 2014; 23(2):366-375.

2. Organização das Nações Unidas (ONU). Transformando nosso mundo: A Agenda 2030 para o Desenvolvimento Sustentável. 2015. [acessado 2020 nov 03]. Disponível em: https://brasil.un.org/

3. FAO. Marco de la FAO para la Agenda Alimentaria Urbana. Roma: FAO; 2019.

4. Barreto LM. Desigualdades em Saúde: uma perspectiva global. Cien Saude Colet 2020; 22(7):2097-2108.

5. Machado CV, Conill EM, Lobato LVC. International context and national policies: challenges facing social protection and health systems in a changing [editorial]. Cien Saude Colet 2018; 23(7):2078-2078.

6. Moreira MR, Kastrup E, Ribeiro JM, Carvalho AI, Braga AP. O Brasil rumo a 2030? Percepções de especialistas brasileiros(as) em saúde sobre o potencial de o País cumprir os ODS. Saude Debate 2019; 43(n. spe 7):22-35.

7. FAO, FIDA, OMS, PMA y UNICEF. Versión resumida de El estado de la seguridad alimentaria y la nutrición en el mundo 2020. Transformación de los sistemas alimentarios para que promuevan dietas asequibles $y$ saludables. Roma: FAO; 2020.

8. Santos BS. A Cruel Pedagogia do Vírus. Coimbra: Almedina; $2020.32 \mathrm{p}$

9. Horton R. Offline: COVID-19 is not a pandemic. The Lancet 2020 Correspondence; 396:874.

10. Turner II BL, Kasperson RE, Matson PA, McCarthy JJ, Corell RW, Christensen L, Eckley N, Kasperson JX, Luers A, Martello ML, Polsky C, Pulsipher A, Schiller A. "A Framework for Vulnerability Analysis in Sustainability Science", Proceedings of the National Academy of Sciences of the United States of America. 2003; 100(14):8074-8079.

11. Singer M, Clair S. Syndemics and Public Health: Reconceptualizing Disease in Bio-Social Context. Medical Anthropology Quarterly 2003; 17(4):423-441.

12. Swinburn BA, Kraak VI, Allender S, Atkins VJ, Baker PI, Bogard JR, Brinsden H, Calvillo A, De Schutter O, Devarajan R, Ezzati M, Friel S, Goenka S, Hammond RA, Hastings G, Hawkes C, Herrero M, Hovmand PS, Howden M, Jaacks LM, Kapetanaki AB, Kasman M, Kuhnlein HV, Kumanyika SK, Larijani B, Lobstein T, Long MW, Matsudo VKR, Mills SDH, Morgan G, Morshed A, Nece PM, Pan A, Patterson DW, Sacks G, Shekar M, Simmons GL, Smit W, Tootee A, Vandevijvere S, Waterlander WE, Wolfenden L, Dietz WH. The Global Syndemic of Obesity, Undernutrition, and Climate Change: The Lancet Commission report. The Lancet 2019; 393:791-846.

13. HLPE. Las perdidas y el desperdício de alimentos en el contexto de sistemas alimentarios sostenibles. Un informe del Grupo de alto nivel de expertos en seguridade alimentaria y nutrición del Comité de Seguridad Alimentaria Mudial. Roma; 2014.

14. Chonchol J. O Desafio Alimentar: a fome no mundo. São Paulo: Marco Zero; 1989. 
15. Brasil. Decreto-Lei n. 11.346, de 15 de setembro de 2006. Dispõe sobre a criação do Sistema Nacional de Segurança Alimentar e Nutricional - SISAN com vistas em assegurar o direito humano à alimentação adequada e dá outras providências. Diário Oficial da União, Brasília, 18 set. 2006.

16. Almeida DAO, Araujo, ML, Ornelas, GM. Agroecologia e sistemas alimentares em cidades-regiões: reflexões acerca de redes internacionais e redes na Região Metropolitana de Belo Horizonte. Cadernos de Agroecologia 2020; 15(2).

17. ONU. Consejo de Derechos Humanos. "La agroecología y el derecho a la alimentación", informe del Relator Especial sobre el derecho a la alimentación, Sr. Olivier De Schutter. 2010, 24p. A/HRC/16/49. [Acceso 2020 Nov 02]. Disponible en: http://www.srfood.org/es/ informes-oficiales

18. Burigo AC, Porto MFS. Trajetórias e aproximações entre a saúde coletiva e a agroecologia. Saude Debate 2019; 43(n. spe 8):248-262.

19. Francis C, Lieblein G, Gliessman S, Breland TA, Creamer N, Harwood R, Salomonsson L, Helenius J, Rickerl D, Salvador R, Wiedenhoeft M, Simmons S, Allen P, Altieri M, Flora C, Poincelot R. Agroecology: the ecology of food systems. J Sust Agric 2003; 22(3):99-118.

20. Gliessman SR. La agroecología - Un movimiento global para la seguridad y la soberanía alimentaria. In: Agroecología para la Seguridad Alimentaria y Nutricional. Actas del Simposio Internacional de la FAO. Roma, Itália: FAO; 2014.

21. IPES-Food. Unravelling the Food-Health Nexus: Addressing practices, political economy, and power relations to build healthier food systems. La Global Alliance for the Future of Food y IPES-Food. 2017.

22. IPES-Food. From uniformity to diversity: a paradigm shift from industrial agriculture to diversified agroecological systems. International Panel of Experts on Sustainable Food systems. 2016.

23. Fundo de Emergência Internacional das Nações Unidas para a Infância (UNICEF). Crianças, alimentação e nutrição: crescendo saudável em um mundo em transformação. Sumário Executivo. Situação Mundial da Infância. 2019.

24. Willett W, Rockström J, Loken B, Springmann M, Lang T, Vermeulen S, Garnett T, Tilman D, DeClerck F, Wood A, Jonell M, Clark M, Gordon LJ, Fanzo J, Hawkes C, Zurayk R, Rivera JA, De Vries W, Sibanda LM, Afshin A, Chaudhary A, Herrero M, Agustina R, Branca F, Lartey A, Fan S, Crona B, Fox E, Bignet V, Troell M, Lindahl T, Singh S, Cornell SE, Reddy KS, Narain S, Nishtar S, Murray CJL. Food in the anthropocene: the EAT-Lancet Commission on healthy diets from sustainable food systems. The Lancet 2019; 393(10170): 447-492.

25. HLPE. La nutrición y los sistemas alimentarios. Un informe del Grupo de alto nivel de expertos en seguridad alimentaria y nutrición del Comité de Seguridad Alimentaria Mundial. Roma; 2017.
26. Ploeg JDVD. Camponeses e impérios alimentares: lutas por autonomia e sustentabilidade na era da globalização. Porto Alegre: UFRGS Editora; 2008.

27. Intergovernmental Panel on Climate Change (IPCC). El cambio climático y la tierra. Informe especial del IPCC sobre el cambio climático, la desertificación, la degradación de las tierras, la gestión sostenible de las tierras, la seguridad alimentaria y los flujos de gases de efecto invernadero en los ecosistemas terrestres. Resumen para responsables de políticas. Genebra: IPCC; 2020.

28. Intergovernmental Science-Policy Platform on Biodiversity and Ecosystem Services (IPBES). Summary for policymakers of the global assessment report on biodiversity and ecosystem services of the Intergovernmental Science-Policy Platform on Biodiversity and Ecosystem Services (IPBES). Bonn, Germany: IPBE; 2019. 56 pp. [cited 2020 Nov 03]. Available from: https://ipbes.net/ global-assessmentreport-biodiversity-ecosystem-services.

29. McMichael P. Regimes Alimentares e Questões Agrárias. São Paulo: Editora Unesp e Editora UFRG; 2016. 256p.

30. High Level Panel of Experts (HLPE). Enfoques agroecológicos y otros enfoques innovadores en favor de la sostenibilidad de la agricultura y los sistemas alimentarios que mejoran la seguridad alimentaria y la nutrición. Un informe del Grupo de alto nivel de expertos en seguridad alimentaria y nutrición del Comité de Seguridad Alimentaria Mundial. Roma: HLPE; 2019.

31. FAO. Los 10 elementos de la agroecología. Guía para la transición hacia sistemas alimentarios y agrícolas sostenibles. FAO; 2018. [acceso 2020 Nov 02]. Disponible en: http://www.fao.org/3/i9037es/I9037ES.pdf

32. Instituto Brasileiro de Geografia e Estatística (IBGE). Censo Agropecuário 2017. Resultados definitivos. [acessado 2020 nov 03]. Disponível em: https://censos. ibge.gov.br/agro/2017/resultados-censo-agro-2017. html

33. Peres J, Matioli V. Donos do Mercado. Como os grandes supermercados exploram trabalhadores, fornecedores e a sociedade. São Paulo: Editora Elefante; 2020.

34. Organização Pan-Americana da Saúde (OPAS). Sistemas alimentares e nutrição: a experiência brasileira para enfrentar todas as formas de má nutrição. Brasília: OPAS; 2017.

35. Rede PENSSAN. Insegurança alimentar e Covid-19 no Brasil. Rede Brasileira de Pesquisa em Soberania e Segurança Alimentar e Nutricional. Relatório de pesquisa. 2021. [acessado 2020 nov 03]. Disponível em: http:// olheparaafome.com.br/

36. Fakhri M, Elver H, De Schutter O. The UN food systems summit: how not to respond to the urgency of reform. New York; 22 mar 2021. [cited 2020 Nov 03]. Available from: http://www.ipsnews.net/2021/03/unfood-systems-summit-not-respond-urgency-reform/

37. Viana P. Mapeamento de experiências em comida de verdade destaca aprendizados essenciais sobre o abastecimento alimentar no Brasil. Ação Coletiva Comida de Verdade. [acessado 2020 nov 03]. Disponível em: https://acaocoletivacomidadeverdade.org/ 
38. Articulação Nacional de Agroecologia (ANA). Municípios agroecológicos e politicas de futuro: iniciativas municipais de apoio à agricultura familiar e à agroecologia e de promoção da segurança alimentar e nutricional. Londres F, Monteiro D, Brochardt V, Maselli $\mathrm{M}$, Jomalinis E, organizadores. Rio de Janeiro: ANA; 2021.

Artigo apresentado em 20/11/2020

Aprovado em 28/06/2021

Versão final apresentada em 30/06/2021

Editores-chefes: Romeu Gomes, Antônio Augusto Moura da Silva 